\title{
A mathematical analysis of the motion of an in-flight soccer ball
}

\section{None given}

Received: date / Accepted: date

\begin{abstract}
In this paper an analytical and numerical study of the three-dimensional equations describing the motion through the air of a spinning ball is presented. The initial analysis involves constant drag coefficients but is later extended to involve drag varying with the spin ratio. Excellent agreement is demonstrated between numerical and analytical results. The analytical solution shows explicitly how the balls motion depends on parameters such as ball roughness, velocity and atmospheric conditions. The importance of applying three-dimensional models, rather than two-dimensional approximations, is demonstrated.
\end{abstract}

Keywords aerodynamics $\cdot$ soccer ball flight $\cdot$ spinning soccer ball $\cdot$ Magnus force $\cdot$ perturbation solution

\section{Introduction}

Over the years much work has been carried out on football design, moving from the traditional leather ball encasing an inflated pigs bladder to the most recent Jabulani which is constructed using polyurethane panels. The goal of the technological advances in football design has been to make the ball more reliable and better to play with, for the benefit of all players involved. Recently, at a mathematics in industry study group held in Johannesburg, the coach of a South African premiership team, Bidvest Wits, posed the question of whether a match ball could be chosen to benefit the home side (or alternatively disadvantage the visiting team). This paper has evolved from that study and in the conclusions the question of whether the ball choice can indeed provide a home advantage will be discussed.

In professional football matches in South Africa, and many other countries, the home team provides the ball. The choice of ball is of course restricted, primarily by Fifa law 2, which dictates the size, (dry) weight, pressure and

Address(es) of author(s) should be given 
material. Hence the main changes in ball design have entered through the material and shape of the panels [10]. A further restriction comes through the sponsor, in that the team must use one of their balls. In the case of Bidvest Wits the balls are provided by Nike. During the meeting in Johannesburg three Nike balls were presented, the balls had a very similar appearance, however there was one significant difference in that two balls were rough (with dimpled panels) while one ball had smooth panels (see, for example, close-up pictures of the Nike T90 Tracer and Catalyst on Nike.com).

To answer the question on the choice of ball the following analysis will focus on its motion through the air and in particular from a free kick or corner. One reason for this is that these are relatively controlled situations and there is much data on a ball's motion through the air. A second reason is that free kicks are an important factor in scoring: in the 1998 world cup 42 of the 171 goals scored came from set-plays, with $50 \%$ of these from free kicks [2]. Consequently understanding the ball motion through the air from a free kick or corner could provide important information concerning the best ball choice. A final reason is that Johannesburg is located high above sea level (at around $1800 \mathrm{~m}$ ). The air density is approximately $20 \%$ lower than that at sea level [11] and so it is expected that the greatest difference in ball motion between Johannesburg and cities located at a lower altitude will be when it moves through the air.

Of course there has been much work carried out on the motion of balls and projectiles through the air. As early as the $1600 \mathrm{~s}$ Newton noted that a spinning tennis ball had a curved trajectory. A century later Robin showed there was a transverse aerodynamic force on a rotating sphere: this is what is now termed the Magnus effect (or more fairly the Robin-Magnus effect). In the late 1800 s Tait was the first to apply this notion to a sports ball (unfortunately a golfball rather than a football). All of this work involved frictionless fluids and so the correct explanation for the Magnus effect had to wait until Prandtl's boundary layer theory of 1904. Specific analysis of the motion of an in-flight football came much later. Asai et al. [1] carried out an experimental study of the lift coefficients on a spinning and non-spinning football and showed a strong dependence on the lift coefficient with spin rate. The equations of motion for a football are provided in many papers, however, the convention of Goff and Carré [7] is used in this study, where the Magnus effect is split into lift and lateral components (Asai et al. also work with two components). Hörzer et al. [11] provide useful details of the differences in atmospheric conditions between World Cup venues in South Africa and demonstrate the effect of altitude on a ball's trajectory. Oggiano and Sætran [14] compare the trajectory of different footballs: this confirmed the belief that the ball choice could provide a home advantage.

The organisation of this paper is as follows. In $\S 2$ the three-dimensional mathematical model is given, this is well established and simply involves Newton's second law. Due to the nonlinearity of the governing equations all previous solutions have been obtained numerically. In an analysis of a simpler model than that considered in the present study, Bray and Kerwin [2] state that the 
governing equations have no closed form solution, although this is technically correct an accurate approximate solution is derived in $\S 3$. This clearly shows the effect of the model parameters on the ball's motion, which is not possible with a numerical solution. In $\S 4$ numerical solutions are calculated to verify the accuracy of the approximate solution. Experimental studies on drag and lift coefficients have demonstrated their dependence on spin, however theoretical studies generally set the coefficients as constant. In $\S 5$ the analysis is extended to deal with variable coefficients. Finally, the question of whether the choice of ball can really provide a home advantage is discussed.

\section{Mathematical model}

The motion of a football is described by Newton's second law $\mathbf{F}=m \ddot{\mathbf{x}}$, where the force $\mathbf{F}$ is comprised of gravity and drag due to the air. For a non-spinning ball the drag force acts solely in the direction opposite to the ball's motion. For a spinning ball the drag has two components, the resistance opposing forward motion and the Magnus force. The Magnus force acts perpendicular to the direction of motion and the spin axis. This force may be resolved into two components denoting lift and lateral motion, see $[1,7]$. If the ball moves in the direction defined by the velocity vector $\hat{\boldsymbol{v}}$ (where the hat indicates a unit vector) then a second vector $\hat{\boldsymbol{l}}$ may be defined which is perpendicular to $\hat{\boldsymbol{v}}$ but remains in the same plane as $\hat{\boldsymbol{v}}$ and the gravity vector, $\hat{\mathrm{g}}$. Note that the $z$ component of $\hat{\boldsymbol{l}}$ is positive (i.e. it points upwards). A right handed system is then defined through a third vector $\hat{\boldsymbol{l}} \times \hat{\boldsymbol{v}}$. The co-ordinate system and vectors are shown in Figure 1. So the drag force opposing motion, $\mathbf{F}_{d}$, acts in the direction $-\hat{\boldsymbol{v}}$, the lift $\mathbf{F}_{l}$ acts in the $\hat{\boldsymbol{l}}$ direction and the lateral force $\mathbf{F}_{s}$ acts in the $\hat{\boldsymbol{l}} \times \hat{\boldsymbol{v}}$ direction. The total force is now defined as

$$
\mathbf{F}=m \mathbf{g}+\mathbf{F}_{d}+\mathbf{F}_{l}+\mathbf{F}_{s}
$$

Splitting the spin components requires two spin speeds to be defined: $\omega_{l}$ is the spin rate about the $\hat{\boldsymbol{l}}$ axis and $\omega_{s}$ is the spin rate about the $\hat{\boldsymbol{l}} \times \hat{\boldsymbol{v}}$ axis. If a single spin axis is used (for example as defined in [11]), which is contained within the $\hat{\boldsymbol{l}}, \hat{\boldsymbol{l}} \times \hat{\boldsymbol{v}}$ plane, then the axis makes an angle $\Omega$ with the $\hat{\boldsymbol{l}} \times \hat{\boldsymbol{v}}$ axis, where $\tan \Omega=\omega_{l} / \omega_{s}$. Note, the current problem is more general than that studied in [2] where the spin axis is contained solely in the $x-z$ plane.

The signs of the forces depend on the convention used. The drag force $\mathbf{F}_{d}$ of course acts in the opposite direction to the ball's forward motion. If pure side-spin is considered, then the $z$ axis is the spin axis. Using standard polar co-ordinate notation as shown in Figure 1 , with $\psi$ contained in the $x-y$ plane and measured from the $x$ axis, and defining $\dot{\psi}=\omega_{s}$, then the resultant force $F_{s}$ acts in the negative $x$ direction. Pure top-spin has the $x$ axis as the spin axis and then $\dot{\theta}=\omega_{l}$ and the resultant force $F_{l}$ acts in the negative $z$ direction. 


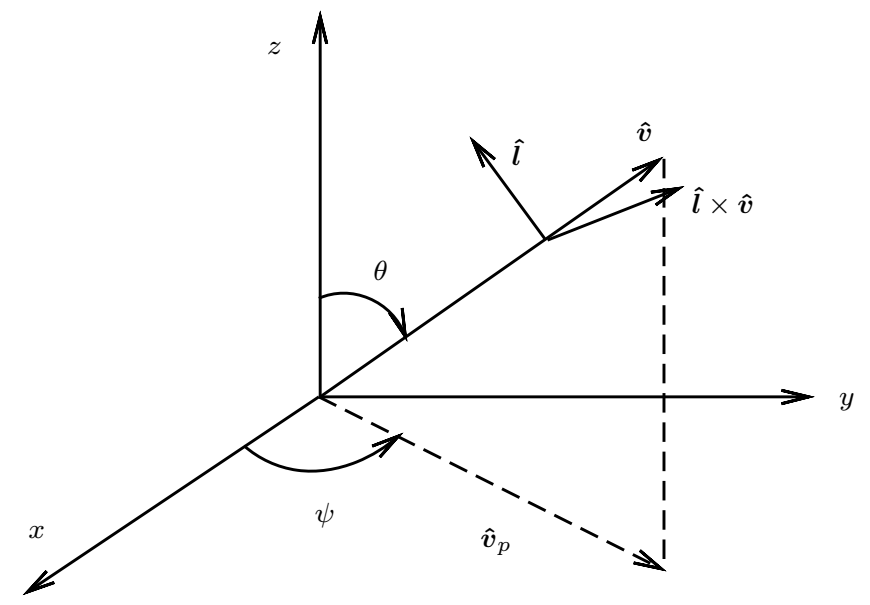

Fig. 1 Co-ordinate system.

Consequently the following relationships hold:

$$
\mathbf{F}_{d}=-\frac{1}{2} \rho A|\boldsymbol{v}|^{2} C_{d} \hat{\boldsymbol{v}}, \quad \mathbf{F}_{l}=-\frac{1}{2} \rho A|\boldsymbol{v}|^{2} C_{l} \hat{\boldsymbol{l}}, \quad \mathbf{F}_{s}=\frac{1}{2} \rho A|\boldsymbol{v}|^{2} C_{s} \hat{\boldsymbol{\imath}} \times \hat{\boldsymbol{v}} .
$$

Here $m$ is the mass of the ball, $A$ is its cross-sectional area, $\rho$ is the density of air, $C_{i}$ are the drag coefficients and

$$
|\boldsymbol{v}|=\sqrt{\left(\dot{x}^{2}+\dot{y}^{2}+\dot{z}^{2}\right)}
$$

where $\boldsymbol{v}=(\dot{x}, \dot{y}, \dot{z})$ is the velocity vector. The product $\hat{\boldsymbol{l}} \times \hat{\boldsymbol{v}}$ acts in the negative $x$ direction so there is no minus sign in the expression for $\mathbf{F}_{s}$. The drag coefficients $C_{i}$ vary with $R e=\rho|\boldsymbol{v}| D / \mu$ (where $D$ is the ball diameter and $\mu$ the air viscosity) and the spin parameter $S p=D \omega /(2|\boldsymbol{v}|)[1,4]$. The variation of $C_{d}$ with Re shows a radical change as the ball moves through the laminar-turbulent transition. The post-transition experimental results of [1] indicate $C_{d}, C_{l}$ vary with $S p$ but have little variation with $R e$, see also [7]. Note, since $C_{l}, C_{s}$ arise from the same effect they have the same behaviour. Since the Magnus force is resolved into two components there are two angular velocities, $\omega_{l}, \omega_{s}$. Then the variation of $C_{l}, C_{s}$ with $S p$ means that they take different values if $\omega_{l} \neq \omega_{s}$. The advantage of using two drag coefficients is that the different spin axes may be differentiated easily. In particular, for pure side-spin $\omega_{l}, C_{l}=0$ and for pure top-spin $\omega_{s}, C_{s}=0$ (the relation between $\omega$ and $C$ will be discussed in more detail later). 
The unit vectors may be defined in terms of the two angles $\psi, \theta$ by the following relations

$$
\begin{aligned}
& \hat{\boldsymbol{v}}=\sin \theta \cos \psi \mathbf{i}+\sin \theta \sin \psi \mathbf{j}+\cos \theta \mathbf{k} \\
& \hat{\boldsymbol{l}}=-\cos \theta \cos \psi \mathbf{i}-\cos \theta \sin \psi \mathbf{j}+\sin \theta \mathbf{k} \\
& \hat{\boldsymbol{l}} \times \hat{\boldsymbol{v}}=-\sin \psi \mathbf{i}+\cos \psi \mathbf{j} .
\end{aligned}
$$

Alternatively the angles in terms of the Cartesian velocity components can be expressed as

$$
\sin \theta=\frac{\left|\boldsymbol{v}_{p}\right|}{|\boldsymbol{v}|}, \quad \cos \theta=\frac{\dot{z}}{|\boldsymbol{v}|}, \quad \sin \psi=\frac{\dot{y}}{\left|\boldsymbol{v}_{p}\right|}, \quad \cos \psi=\frac{\dot{x}}{\left|\boldsymbol{v}_{p}\right|},
$$

where $\boldsymbol{v}_{p}=(\dot{x}, \dot{y})$ is the projection of $\boldsymbol{v}$ on the $x-y$ plane (see Figure 1).

The equation of motion may now be written in component form

$$
\begin{aligned}
& \ddot{x}=-k_{d}|\boldsymbol{v}| \dot{x}+k_{l} \frac{|\boldsymbol{v}|}{\left|\boldsymbol{v}_{p}\right|} \dot{x} \dot{z}+k_{s} \frac{|\boldsymbol{v}|^{2}}{\left|\boldsymbol{v}_{p}\right|} \dot{y}, \\
& \ddot{y}=-k_{d}|\boldsymbol{v}| \dot{y}+k_{l} \frac{|\boldsymbol{v}|}{\left|\boldsymbol{v}_{p}\right|} \dot{y} \dot{z}-k_{s} \frac{|\boldsymbol{v}|^{2}}{\left|\boldsymbol{v}_{p}\right|} \dot{x}, \\
& \ddot{z}=-g-k_{d}|\boldsymbol{v}| \dot{z}-k_{l}|\boldsymbol{v}|\left|\boldsymbol{v}_{p}\right| .
\end{aligned}
$$

The scaled drag coefficients are $k_{i}=\rho A C_{i} /(2 m)$. This is a nonlinear set of second order ordinary differential equations with no analytical solution. They may easily be solved numerically however analytical progress can be made after applying certain sensible assumptions concerning the ball's motion:

1. The axes can be chosen so that the ball is kicked primarily in one direction (although due to swerve it will obviously deviate from this). Hence it is assumed that the ball is kicked mainly in the $y$ direction.

2. The dominant swerve is in the sideways direction, that is, the ball is kicked mainly with side-spin.

The first assumption means that the velocity in the $y$ direction must be significantly higher than that in the $x$ and $z$ directions, $\dot{y} \gg \dot{x}, \dot{z}$ and also the ball travels furthest in the $y$ direction. The second assumption holds in general for football motion, side-spin is usually greater than top-spin, for a golf ball one would expect a greater top-spin. The analysis is easily adapted to top-spin but, since the focus is on footballs, dominant side-spin is assumed.

\section{Perturbation solution}

To make analytical progress the system is non-dimensionalised by setting

$$
X=\frac{x}{L_{1}}, \quad Y=\frac{y}{L_{2}}, \quad Z=\frac{z}{L_{3}}, \quad T=\frac{t}{\tau} .
$$




\begin{tabular}{|c|c|c|c|c|c|}
\hline$k_{d}$ & 0.013 & & $k_{l}$ & 0.004 & \\
$k_{s}$ & 0.0108 & & $m$ & 0.45 & $\mathrm{~kg}$ \\
$\rho$ & 1 & $\mathrm{~kg} \mathrm{~m}^{-3}$ & $D$ & 0.22 & $\mathrm{~m}$ \\
$A$ & 0.039 & $\mathrm{~m}^{2}$ & $v$ & 25 & $\mathrm{~m} / \mathrm{s}$ \\
$C_{d}$ & 0.3 & & $C_{s}$ & 0.25 & \\
$C_{l}$ & 0.1 & & $L_{2}$ & 20 & $\mathrm{~m}$ \\
\hline
\end{tabular}

Table 1 Typical parameter values, see [1,5,15]

where $L_{2}$ is the distance in the $y$ direction to the goal, $\tau=L_{2} / v$ is the timescale for the ball to reach the target where $v$ is the initial velocity of the ball in the $y$ direction and $L_{3}=g \tau^{2}$ is the vertical length-scale. The capital letters $X, Y, Z, T$ denote non-dimensional variables. The length-scale $L_{1}$ is, as yet, unknown but it is noted that due to assumption $1, L_{1} \ll L_{2}$.

First, consider the velocity vectors. Writing them in terms of non-dimensional quantities

$|\boldsymbol{v}|=\frac{L_{2}}{\tau} \dot{Y} \sqrt{1+\frac{L_{1}^{2}}{L_{2}^{2}} \frac{\dot{X}^{2}}{\dot{Y}^{2}}+\frac{L_{3}^{2}}{L_{2}^{2}} \frac{\dot{Z}^{2}}{\dot{Y}^{2}}}=\frac{L_{2}}{\tau} \dot{Y} r_{1}, \quad\left|\boldsymbol{v}_{p}\right|=\frac{L_{2}}{\tau} \dot{Y} \sqrt{1+\frac{L_{1}^{2}}{L_{2}^{2}} \frac{\dot{X}^{2}}{\dot{Y}^{2}}}=\frac{L_{2}}{\tau} \dot{Y} r_{2}$,

where $r_{1}, r_{2}$ denote the square roots. The factor $L_{2} \dot{Y} / \tau$ has been isolated since this is the largest term in the velocity expression and so $r_{1}, r_{2}=\mathcal{O}(1)$ (that is their size is of the order unity).

The governing equations may now be written

$$
\begin{aligned}
& \ddot{X}=-k_{d} L_{2} \dot{X} \dot{Y} r_{1}+k_{l} L_{3} \frac{r_{1}}{r_{2}} \dot{X} \dot{Z}+k_{s} \frac{L_{2}^{2} r_{1}^{2}}{L_{1} r_{2}} \dot{Y}^{2} \\
& \ddot{Y}=-k_{d} L_{2} \dot{Y}^{2} r_{1}+k_{l} L_{3} \frac{r_{1}}{r_{2}} \dot{Y} \dot{Z}-k_{s} \frac{L_{1} r_{1}^{2}}{r_{2}} \dot{X} \dot{Y} \\
& \ddot{Z}=-1-k_{d} L_{2} r_{1} \dot{Y} \dot{Z}-k_{l} \frac{L_{2}^{2}}{L_{3}} r_{1} r_{2} \dot{Y}^{2} .
\end{aligned}
$$

To determine $L_{1}$ it is noted that the motion in the $x$ direction is caused by either an initial velocity or the spin component in the $y$ direction. Since this study is focussed on the spin induced swerve, the initial velocity in the $x$ direction is set to zero, $\dot{x}(0)=\dot{X}(0)=0$. The dominant term in equation (13) is clearly that involving $\dot{Y}^{2}$ and since the velocity vectors are scaled so that $r_{1}, r_{2}=\mathcal{O}(1), L_{1}=k_{s} L_{2}^{2}$. Using the parameter values of Table 1 this determines $L_{1} \approx 4 \mathrm{~m}$, that is, the ball is expected to travel of the order $4 \mathrm{~m}$ laterally during its flight. The dominant term in equation (14) again involves $\dot{Y}^{2}$. With $k_{d}=0.013, k_{d} L_{2}=0.26$ and this is denoted as $\epsilon=k_{d} L_{2}$ which is a small parameter (and $\epsilon^{2} \approx 0.07$ ). The square root terms $r_{1}, r_{2}$ contain the ratios $L_{1}^{2} / L_{2}^{2} \approx 0.04, L_{3}^{2} / L_{2}^{2} \approx 0.25$ which are denoted $c_{1} \epsilon^{2}, c_{2} \epsilon$ respectively (note, $k_{s} L_{1}=L_{1}^{2} / L_{2}^{2}$ ). Finally, $k_{l} L_{3} \approx 0.03=c_{3} \epsilon^{2}, k_{l} L_{2}^{2} / L_{3} \approx 0.27=c_{4} \epsilon$. 
The governing equations are now

$$
\begin{aligned}
& \ddot{X}=-\dot{Y} r_{1}\left[\epsilon \dot{X}-c_{3} \epsilon^{2} \frac{\dot{X} \dot{Z}}{r_{2} \dot{Y}}-\frac{r_{1}}{r_{2}} \dot{Y}\right] \\
& \ddot{Y}=-\dot{Y} r_{1}\left[\epsilon \dot{Y}-c_{3} \epsilon^{2} \frac{\dot{Z}}{r_{2}}+c_{1} \epsilon^{2} \frac{r_{1}}{r_{2}} \dot{X}\right] \\
& \ddot{Z}=-1-\dot{Y} r_{1}\left[\epsilon \dot{Z}+c_{4} \epsilon r_{2} \dot{Y}\right]
\end{aligned}
$$

where

$$
r_{1}=\sqrt{1+c_{1} \epsilon^{2} \frac{\dot{X}^{2}}{\dot{Y}^{2}}+c_{2} \epsilon \frac{\dot{Z}^{2}}{\dot{Y}^{2}}}, \quad r_{2}=\sqrt{1+c_{1} \epsilon^{2} \frac{\dot{X}^{2}}{\dot{Y}^{2}}} .
$$

Note the assumption that the ball is kicked primarily with side-spin means that the $k_{l}$ terms enter at lower order to the $k_{s}$ terms in the $\ddot{X}, \ddot{Y}$ equations (through the coefficient $c_{3}$ ). In the $\ddot{Z}$ equation lift is the dominant force after gravity and so it enters at $\mathcal{O}(\epsilon)$. With top-spin dominating the scaling would have to be changed accordingly.

Written in non-dimensional form observations can be made about the solution behaviour, without solving the system. For example, all terms in (17) involve the small parameter $\epsilon$ indicating that the dominant motion in the $Y$ direction is described by $\ddot{Y}=0$ and that drag, represented by $\epsilon$, has a relatively small effect. Since $\epsilon=0.26$ neglecting terms involving $\epsilon$ could lead to errors of around $26 \%$. Motion in the $Z$ direction is dominated by gravity. The $X$ motion is dominated by the contribution of $\dot{Y}$ (reflecting the fact that the Magnus force is due to the difference in equatorial velocities and the forward velocity, which is approximately $\dot{Y})$. However, the most interesting feature is that since $r_{1}$ contains the term $\epsilon(\dot{Z} / \dot{Y})^{2}$ the velocity in the $Z$ direction will contribute to all equations. Most importantly it will affect the $X$ equation at order $\epsilon$, i.e. for the current set of parameter values neglect of the $Z$ motion may lead to errors of around $26 \%$. Obviously this could have significant consequences for any experiment using a two-dimensional analysis to model the three dimensional ball motion. This is discussed further in the results section, $\S 4$.

The initial conditions are that the ball starts at the origin and is kicked with (dimensional) velocity $(0, v, w)$. Note, the velocity in the $x$ direction could always be chosen to be zero by simply defining the $y$ axis as the direction of the kick in the $x-y$ plane. In non-dimensional form these conditions are $X(0)=Y(0)=Z(0)=0, \dot{X}(0)=0, \dot{Y}(0)=1, \dot{Z}(0)=W$, where $W=w \tau / L_{3}$.

In order to determine a standard perturbation solution based on the small parameter $\epsilon$ write

$$
X=X_{0}+\epsilon X_{1}+\epsilon^{2} X_{2}+\cdots, \quad Y=Y_{0}+\epsilon Y_{1}+\epsilon^{2} Y_{2}+\cdots, \quad Z=Z_{0}+\epsilon Z_{1}+\epsilon^{2} Z_{2}+\cdots
$$


To $\mathcal{O}\left(\epsilon^{2}\right)$ the solution is

$$
\begin{aligned}
X= & \frac{T^{2}}{2}+\epsilon\left[\frac{c_{2} W^{2} T^{2}}{2}-\left(3+2 c_{2} W\right) \frac{T^{3}}{6}+\frac{c_{2} T^{4}}{12}\right]+ \\
& \epsilon^{2}\left[\left(-\frac{9}{2} c_{2} W^{2}+3 c_{3} W-2 c_{2} c_{4} W\right) \frac{T^{3}}{6}+\left(6 c_{2} W+\frac{11}{2}-2 c_{3}-\frac{c_{1}}{2}+2 c_{2} c_{4}\right) \frac{T^{4}}{12}-\frac{13 c_{2} T^{5}}{120}\right] \\
Y= & T-\epsilon \frac{T^{2}}{2}+\epsilon^{2}\left[-\left(c_{2} W^{2}-2 c_{3} W\right) \frac{T^{2}}{4}+\left(2-c_{1}+c_{2} W-c_{3}\right) \frac{T^{3}}{6}-\frac{c_{2} T^{4}}{24}\right] \\
Z= & W T-\frac{T^{2}}{2}+\epsilon\left[-\left(W+c_{4}\right) \frac{T^{2}}{2}+\frac{T^{3}}{6}\right]+ \\
& \epsilon^{2}\left[-c_{2} W^{2}\left(W+c_{4}\right) \frac{T^{2}}{4}+\left(4 W+6 c_{4}+3 c_{2} W^{2}+2 c_{2} c_{4} W\right) \frac{T^{3}}{12}-\left(3+3 c_{2} W+c_{2} c_{4}\right) \frac{T^{4}}{24}+\frac{c_{2} T^{5}}{40}\right] .
\end{aligned}
$$

Bray \& Kerwin [3] study a simplified two-dimensional system. This analysis follows the line of equating the acceleration to the largest term on the right hand side of the equations. From equations $(16,17)$ this reduces the problem to

$$
\ddot{X}=\dot{Y}^{2}, \quad \ddot{Y}=-\epsilon \dot{Y}^{2},
$$

where it is noted that $r_{1} \approx r_{2} \approx 1$. Applying the initial conditions gives

$$
Y=\frac{1}{\epsilon} \ln |1+\epsilon T|, \quad X=-\frac{1}{\epsilon}(Y-T) .
$$

To relate these solutions to equations $(21,22)$ the Taylor series expansion for $\epsilon \ll 1$ is used to find

$$
\begin{aligned}
& X=\frac{T^{2}}{2}-\epsilon \frac{T^{3}}{3}+\epsilon^{2} \frac{T^{4}}{4} \\
& Y=T-\epsilon \frac{T^{2}}{2}+\epsilon^{2} \frac{T^{3}}{3} .
\end{aligned}
$$

Setting $W, c_{i}=0$ in $(21,22)$, to remove the $Z$ dependence and make the solution appear two-dimensional, the $x$ solutions differ at $\mathcal{O}(\epsilon)$, while the $y$ solutions differ at $\mathcal{O}\left(\epsilon^{2}\right)$. The simplification of only taking the largest terms on the right hand side will therefore correctly capture the dominant behaviour but, in the case of the sideways swerve, leads to errors in all subsequent terms. Consequently, this solution form will provide a reasonable approximation to the numerical result for $y$ but a rather poorer one for $x$ and the error increases with $\epsilon$ (for example if $L_{2}$ is increased). Further deviation from the correct 
solution will occur due to the neglect of the $Z$ motion. This will be discussed later in the context of the solutions shown in $\S 4$.

Now return to dimensional variables $X=x / L_{1}, Y=y / L_{2}, Z=z / L_{3}$, $T=t / \tau$, where $L_{1}=k_{s} L_{2}^{2}, L_{3}=g \tau^{2}, \tau=L_{2} / v$ and $L_{2}, v$ are the initial distance and velocity in the $y$ direction. The constants must also be expressed in terms of the dimensional parameters

$\epsilon=k_{d} L_{2}, \quad c_{1}=\frac{k_{s}^{2}}{k_{d}^{2}}, \quad c_{2}=\frac{g^{2} L_{2}}{k_{d} v^{4}}, \quad c_{3}=\frac{k_{l} g}{v k_{d}^{2} L_{2}}, \quad c_{4}=\frac{k_{l} v^{2}}{g k_{d} L_{2}}, \quad W=\frac{w v}{g L_{2}}$.

The dimensional solution is then given by

$$
\begin{aligned}
& x=\frac{k_{s}(v t)^{2}}{2}\left[1-\left\{k_{d} v t-\frac{g^{2} t^{2}-4 g w t+6 w^{2}}{6 v^{2}}\right\}\right] \\
& y=v t\left[1-\left\{\frac{k_{d} v t}{2}\right\}\right] \\
& z=w t-\frac{g t^{2}}{2}+\left\{\frac{k_{d} g v t^{3}}{6}-\left(k_{d} w+k_{l} v\right) \frac{v t^{2}}{2}\right\} .
\end{aligned}
$$

Note, since the expressions are rather cumbersome they are only written here to $\mathcal{O}(\epsilon)$ (the $\mathcal{O}(\epsilon)$ terms are given in the curly brackets). From these expressions the effect of the various parameters can be seen clearly. The $y$ equation shows that the distance travelled is approximately proportional to the initial velocity and time but that drag reduces this and the effect of drag increases with time. In the $z$ direction the distance travelled is determined primarily by the initial velocity and gravity but both drag and lift act to change this. Again these effects increase with time. However, since swerve due to side-spin is the main focus of this study the $x$ equation is the most revealing. From this it is seen that, to leading order the distance travelled in the $x$ direction is proportional to the sideways drag coefficient $k_{s}$ and also $(v t)^{2}$. The ball design and atmospheric conditions contribute through $k_{s}$ hence ball design is very important for swerve. The product $v t$ is the first approximation to the distance travelled in the $y$ direction. The quadratic dependence then indicates the importance of taking a kick far from the goal. The further the ball travels the more it will swerve (this is obviously well known to free kick specialists in football who frequently attempt to move the ball away from the goal). Perhaps the most famous example is the free-kick of Roberto Carlos against France in 1997 which exhibited spectacular curve at the end of the flight. This free kick was taken approximately $35 \mathrm{~m}$ from goal, see YouTube or [6]. The forward drag $k_{d}$ enters at first order and acts to reduce the swerve. An important feature of the solution is that the vertical motion, through $g$ and $w$, also enters at first order. The quadratic $g^{2} t^{2}-4 g w t+6 w^{2}$ is positive provided $w \geq 0$ (i.e. the ball is not kicked into the ground) and so acts to increase the swerve. A two-dimensional analysis will miss this effect and comparison with experiment would then lead to over-prediction of $k_{s}$.

Due to the differences in time dependence of the terms in equations (29-31) they will become less accurate as time increases. This is termed the secular 
effect. The break down will occur when the $\mathcal{O}(\epsilon)$ terms become $\mathcal{O}(1)$. In the case of the $x$ equation this is when $t \sim 1 /\left(k_{d} v\right) \approx 3 \mathrm{~s}$. The $y$ and $z$ equations indicate times around $9 \mathrm{~s}$. Given that the football flight is typically $1 \mathrm{~s}$ these approximations should hold for all sensible kicks the secular effect is not considered.

\section{Numerical and analytical results}
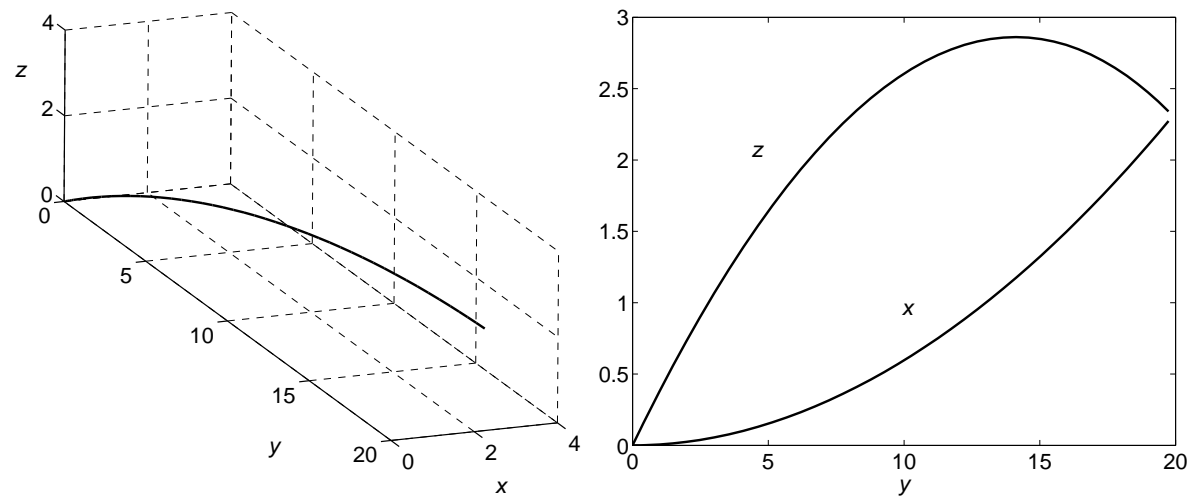

Fig. 2 Left: Numerical solution showing a dimensional trajectory of a soccer ball being hit into the top corner of the goal. Right: plots of $x$ and $z$ against $y$.

The numerical solution to equations (16-18) was carried out using MATLAB routine ODE45. In Figure 2a) the 3D trajectory of a ball kicked with initial conditions $v=25 \mathrm{~m} / \mathrm{s}, w=8.76 \mathrm{~m} / \mathrm{s}$ and at a distance $L_{2}=20 \mathrm{~m}$ from the target is shown. The choice of $w$ was made so that the ball ended up near the top of the goal (since the centre of mass of the ball is tracked the top of the goal is taken at $2.34 \mathrm{~m}$, that is, the standard goal height minus the ball radius). Other parameters are given in Table 1 . To travel $20 \mathrm{~m}$ in the $y$ direction takes $0.9112 \mathrm{~s}$ (without drag $t=20 / 25=0.8 \mathrm{~s}$ ). Figure $2 \mathrm{~b}$ ) demonstrates the $x, z$ variation against $y$. From the $x$ curve it can be seen that the ball moves around $2.3 \mathrm{~m}$, with an approximately quadratic variation. In the $z$ direction the ball height increases until quite close to the goal, reaching a maximum of only $0.4 \mathrm{~m}$ above the goal. To show the trajectory clearly, Figure 2 uses dimensional quantities. Whenever possible, in subsequent figures dimensionless quantities are preferred since these highlight the differences in motion better. They also allow results to be combined, such as in Figure 7, where $x$ and $y$ solutions are plotted on the same graph.

In Figure 3 the variation of $X, Y$ and $Z$ against $T$ is shown, for the same conditions as in Figure 2 but now the various perturbation solutions are included. In non-dimensional units the time of flight is $T=0.9112 \times 25 / 20 \approx$ 

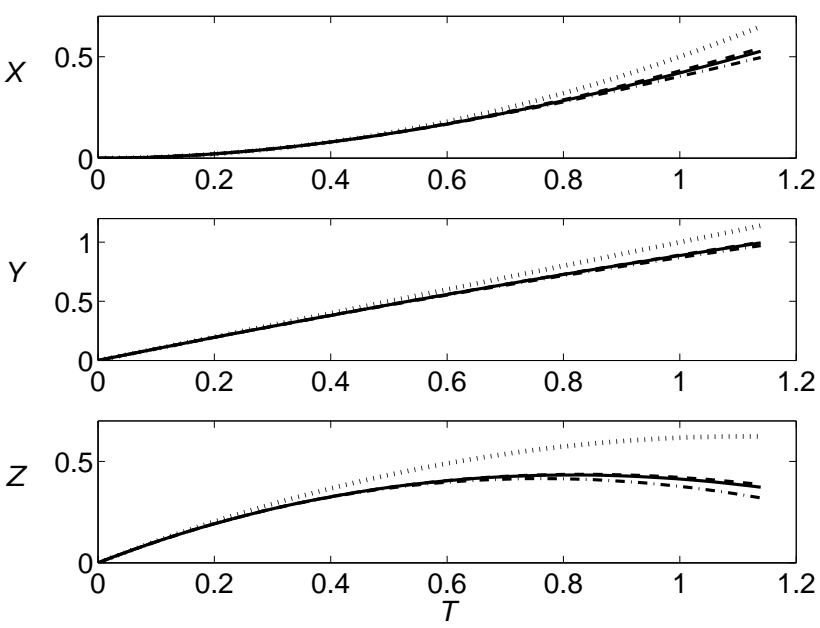

Fig. 3 Non-dimensional trajectories of $X, Y$ and $Z$ against $T$ : full numerical solution (solid line), $\mathcal{O}\left(\epsilon^{0}\right)$ solution (dotted line), $\mathcal{O}(\epsilon)$ solution (dot-dashed line) and $\mathcal{O}\left(\epsilon^{2}\right)$ solution (dashed line). Parameter values are $C_{d}=0.3, C_{s}=0.25$ and $C_{l}=0.1$.

1.14. The solid line is the numerical solution, the dotted line the leading order perturbation, dash-dot is the first order and dashed is the second order. In each case the leading order gives a reasonable approximation for small times but rapidly loses accuracy. To describe the full flight the perturbation solution for $Y$ is reasonably accurate at first order, but the $X$ and $Z$ solutions are less accurate. All curves show excellent agreement at $\mathcal{O}\left(\epsilon^{2}\right)$. Since the second order solution involves neglecting terms $\mathcal{O}\left(\epsilon^{3}\right) \approx 0.02$, errors of the order of $2 \%$ is expected. In fact the error increases with time, obviously at $T=0$ the error is zero due to the imposition of the initial condition. In the $X$ direction the maximum error between the numerical and second order solution is almost $3 \%$ and this occurs at the end of the flight. This is in agreement with the earlier discussion that the approximate solutions start to break down as time increases. Note, the same conclusion holds for the dimensional problem.

In the previous section the apparent problem with neglecting vertical motion was discussed and, in particular, that this would be most apparent in the motion in the $X$ direction. A two-dimensional model, with motion solely in the $X-Y$ plane, is retrieved from this solution by setting $c_{2}=c_{3}=W=0$ in equations $(21,22)$. Figure 4 compares numerical (solid line) and perturbation (dashed line) solutions in $X$ up to second order. The two curves labelled (a) include motion in the $Z$ direction and (b) neglect $Z$ motion. If the $Z$ motion is neglected the swerve is decreased by around $4.5 \%$. This error increases nonlinearly with increasing $w$ : if $w$ is doubled the error increases to $30 \%$. This observation has obvious consequences in the interpretation of experimental studies. For example, if the two-dimensional model of [3] is compared to experiments and used to calculate drag coefficients then to compensate for the 


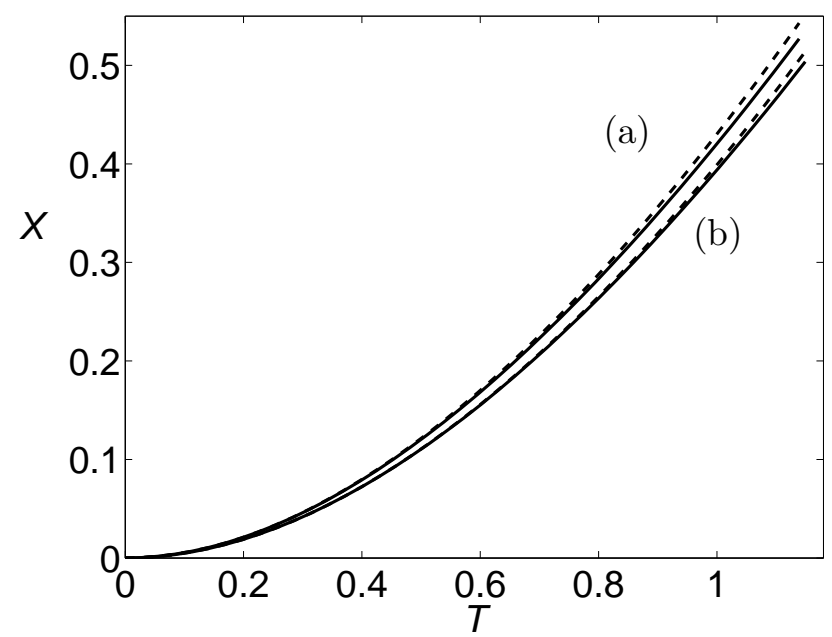

Fig. 4 Comparison of numerical (solid line) and second order perturbation (dashed line) solutions in $X$ : (a) including vertical motion, (b) neglecting vertical motion.

neglect of the $Z$ motion it will predict higher values than in reality. In [2], the mathematical model is simplified by assuming the spin axis remains in the $X-Z$ plane and therefore the $\dot{Z}$ term in equation (16) may be neglected, yet it is retained in the $Y$ equation. This approximation appears rather inconsistent and will also affect predicted drag coefficients. It is therefore important to implement a full three-dimensional model when calculating experimental parameters. A two-dimensional study is possible but, since gravity cannot be avoided, this should be confined to the $Y-Z$ plane and using only top-spin.

\subsection{Comparison with experimental data}

In the paper of Carré et al [5] a number of results are presented for ball motion with and without spin. In their Figures 4a-c a typical trajectory is shown for a ball launched with no spin at approximately $15^{\circ}$ to the ground and with a launch velocity in the range $17-31 \mathrm{~m} / \mathrm{s}$. Taking the data from these figures it is a simple matter to curve fit (in this case the polyfit function of Matlab was used, which fits data using a least-squares criteria). A quadratic fit to the $y(t)$ data of their Figure $4 \mathrm{~b}$ leads to

$$
y \approx a_{0}+a_{1} t+a_{2} t^{2},
$$

where $a_{0}=-0.1785, a_{1}=17.591, a_{2}=-1.237$. A cubic fit to the $z(t)$ data of Figure $4 \mathrm{c}$ leads to

$$
z \approx b_{0}+b_{1} t+b_{2} t^{2}+b_{3} t^{3}
$$


where $b_{0}=0.0253, b_{1}=6.287, b_{2}=-6.294, b_{3}=0.201$. The appropriate data points are displayed on Figure 5 as asterisks. Finally, Figure 4a presents $z(y)$ which takes the form

$$
z \approx c_{0}+c_{1} y+c_{2} y^{2}+c_{3} y^{3}
$$

where $c_{0}=0.008, c_{1}=0.371, c_{2}=-0.023, c_{3}=0.0001$.
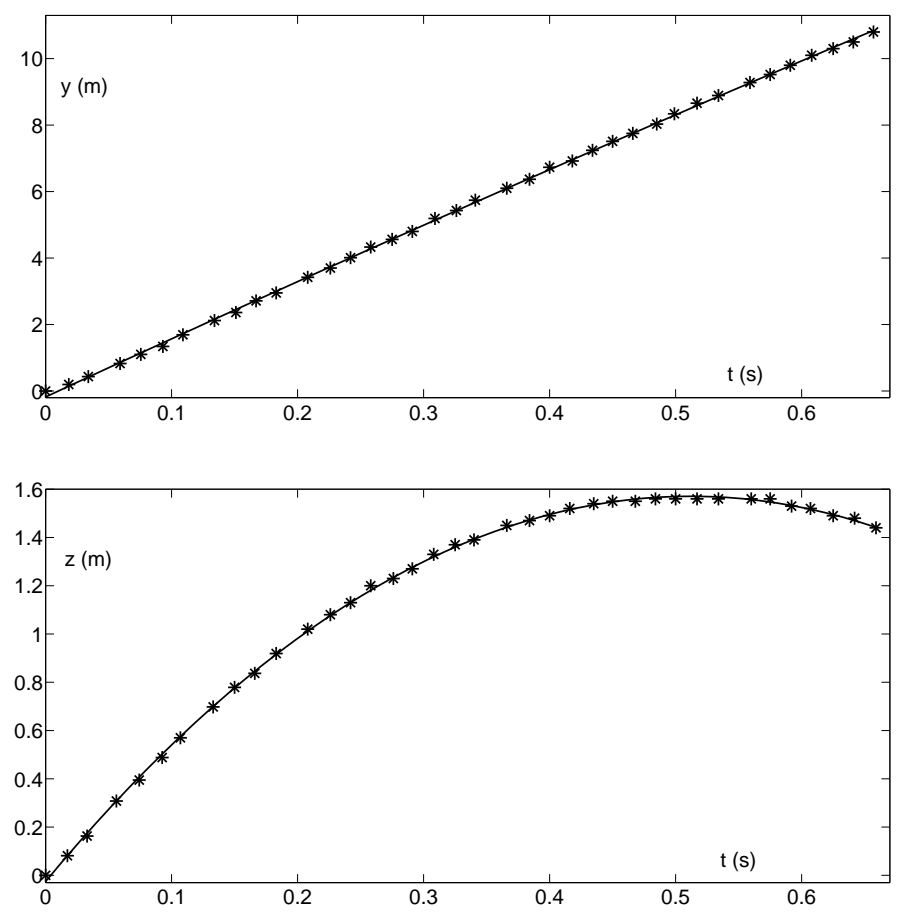

Fig. 5 Comparison of experimental data of [5] (asterisks) and curve fits (solid line)

The initial angle of the kick is easily determined from the curve fits by noting

$$
\tan \theta=\frac{\dot{z}(0)}{\dot{y}(0)}=\frac{b_{1}}{a_{1}} \approx \frac{6.287}{17.591} \approx 0.357 .
$$

Alternatively $\tan \theta=d z /\left.d y\right|_{t=0}=c_{1} \approx 0.371$. These two results indicate a launch angle of $\theta \approx 19.65$ or 20.35 , with an average of approximately $20^{\circ}$ (slightly higher than that quoted in the paper). The initial velocity in the $y, z$ directions is $(\dot{y}(0), \dot{z}(0))=\left(a_{1}, b_{1}\right)=(17.591,6.287)$ and so the initial speed $|\boldsymbol{v}|=\sqrt{a_{1}^{2}+b_{1}^{2}} \approx 18.68 \mathrm{~m} / \mathrm{s}$, which is within the quoted range. 
Comparison of equations $(32,33)$ and $(30,31)$ allows us to determine additional information about the kick. First it may be noted that both $y, z$ should start at the origin, which is the case with the perturbation solution, but the curve fit indicates some slight error. However, both the relevant terms $b_{0}, c_{0}$ are small. The values for $(v, w)$ are obtained from taking $(\dot{y}(0), \dot{z}(0))$ in equations $(30,31)$. The drag coefficient $k_{d}$ may be obtained by comparing the quadratic terms in the $y$ expressions

$$
-\frac{k_{d} v^{2}}{2}=a_{2} \quad \Rightarrow \quad k_{d}=0.008
$$

which is of a similar order of magnitude to that quoted in Table 1 . The $z$ expressions provide a means for verifying this value, comparing the cubic terms gives

$$
\frac{k_{d} g v}{6}=b_{3} \quad \Rightarrow \quad k_{d}=0.007
$$

which, allowing for experimental error and errors in curve fitting, appears to be in good agreement with the first estimate. In [5] it is stated that this trajectory is typical for a case with no-spin, consequently $k_{l}=0$. Comparison of the quadratic terms in the $z$ expressions then determines $k_{d} \approx 0.025$, a result that is clearly at odds with the previous findings. Allowing $k_{l} \neq 0$ the quadratic terms may be used with to determine $k_{l}=0.0061$ when $k_{d}=0.008$ and $k_{l}=0.0065$ when $k_{d}=0.007$.

In Figure 5a) the experimental data is compared against the quadratic form given by equation (32) with the quoted values for $a_{i}$. This is equivalent to plotting the approximate solution (30) with $v=17.591 \mathrm{~m} / \mathrm{s}, k_{d}=0.008$. Clearly the agreement is excellent. In Figure 5b) the experimental data is compared against the cubic form given by equation (33) with the quoted values for $b_{i}$. This is equivalent to plotting the approximate solution (31) with $w=$ $6.287 \mathrm{~m} / \mathrm{s}, k_{d}=0.007$. Clearly the agreement is excellent.

In summary, the forms suggested by the perturbation solution can provide an excellent fit to the experimental data. Comparison between the coefficients of the fitted curve and the perturbation solutions then permits the calculation of the drag coefficients (and can also be used to determine initial velocities if these are unknown). As an example the data provided in [5] was used to determine flight parameters, indicating an initial angle of around $20^{\circ}$, as opposed to the reported $15^{\circ}$, a drag coefficient $k_{d} \in[0.007,0.008]$ and a lift coefficient $k_{l} \in[0.0061,0.0065]$. The appearance of a non-zero lift coefficient for an experiment apparently with no spin is particularly interesting. Given that the equations provide three ways to calculate $k_{d}$ in the absence of spin and that two of these routes agree well it seems likely that the fault does not lie in the equations. Consequently we may infer that possibly the graphs have been misreported (they are in fact only presented as a 'typical' solution) or that some spin was generated during the flight, perhaps due to the initial positioning of the seams or the valve? 


\section{Drag coefficients}

So far the analysis has dealt with constant drag coefficients. However, experimental studies have shown that $C_{d}, C_{l}, C_{s}$ all vary with spin rate $S p=R \omega /|\boldsymbol{v}|$ and, to a lesser extent, (provided the air flow is turbulent) the Reynolds number. During the flight of a ball the angular velocity decays very slowly [11]. This may also be observed in the experimental findings of [9] and so angular velocity may reasonably be taken as constant but the magnitude of $|\boldsymbol{v}|$ decreases due to the drag and so $S p$ increases.

There exist numerous experimental studies showing the variation of $C_{s}$ (or $C_{l}$ ) with $S p$, see $[1,4,9,14]$ for example. Goff and Carré [7] summarise experimental results of Asai et al. [1] and Carré et al. [4]. They point out that since $C_{l}$ and $C_{s}$ arise from the same process they are equivalent (some authors do not split the lift coefficient into components, preferring to work with a single one defined on an appropriate spin axis, see [11] for example). The experimental results indicate that $C_{s}, C_{l}$ increase with spin until levelling off around $S p=0.3$. The actual values vary between experiments and, as shown in [14], with different types of ball but typically range between 0 and 0.35 as $S p$ increases from 0 to $0.3[1,5,9,15]$. Since the focus of this study is on the effect of spin in these calculations the region where $S p$ is very small (below 0.1) is neglected and then a piecewise approximation is employed of the form

$$
C_{s}= \begin{cases}C_{s 0}\left(1+\frac{m_{s}}{C_{s 0}}\left(S p-S p_{0}\right)\right), & 0.1<S p<0.3 \\ C_{s 0}\left(1+\frac{m_{s}}{C_{s 0}}\left(0.3-S p_{0}\right)\right)=C_{s m}, & , S p>0.3\end{cases}
$$

where the gradient $m_{s}=0.8, C_{s 0}=0.25$ is the coefficient when $S p=S p_{0}=$ $0.15 / 0.8$ and $C_{s m}=0.34$ is the constant maximum value of $C_{s}$. The drag coefficient $k_{s}=k_{s 0}$ is now written

$$
k_{s 0}=\frac{\rho A C_{s 0}}{2 m}
$$

The linear form, without the cut-off, has also been employed by Hörzer et al. [11]. The drag coefficient $C_{d}$ shows a weaker dependence on $S p$ and so as a first attempt to model varying coefficients $C_{s}$ is allowed to vary and $C_{d}$ is left constant.

Given the equivalence of $C_{s}, C_{l}$, only the effect of varying one of these quantities is studied. This is fortunate since, as pointed out in the previous section, the analysis should be three-dimensional (unless limited to the $y-z$ plane with only top-spin imposed). If both $C_{s}$ and $C_{l}$ are allowed to vary then the perturbation will be extremely cumbersome. Instead the case of pure sidespin is studied by setting $C_{l}=k_{l}=0$ while $C_{s}$ is given by equation (38). The 
non-dimensional model then reduces to

$$
\begin{aligned}
\ddot{X} & =-\dot{Y} r_{1}\left[k_{d} L_{2} \dot{X}-k_{s 0} \phi_{s} \frac{L_{2}^{2} r_{1}}{L_{1} r_{2}} \dot{Y}\right] \\
\ddot{Y} & =-\dot{Y} r_{1}\left[k_{d} L_{2} \dot{Y}+k_{s 0} \phi_{s} \frac{L_{1} r_{1}}{r_{2}} \dot{X}\right] \\
\ddot{Z} & =-1-k_{d} L_{2} r_{1} \dot{Y} \dot{Z} .
\end{aligned}
$$

where

$$
\phi_{s}=\left\{\begin{array}{l}
1+\frac{m_{s}}{C_{s 0}}\left(S p-S p_{0}\right), 0.1<S p<0.3 \\
\frac{C_{s m}}{C_{s 0}}, \quad S p>0.3
\end{array}\right.
$$

and

$$
S p=\frac{D \omega}{2|\boldsymbol{v}|}=\frac{D \omega}{2 r_{1} v \dot{Y}}
$$

Defining

$$
\phi_{s}=\frac{\phi_{1}}{r_{1} \dot{Y}}+\phi_{2}, \quad \phi_{1}=\frac{m_{s} R \omega}{C_{s 0} v}, \quad \phi_{2}=1-\frac{m_{s} S p_{0}}{C_{s 0}} .
$$

To retrieve the constant spin case the conditions set were $\phi_{1}=0, \phi_{2}=1$. Retaining the previous definitions for the small parameters (but with $k_{s}$ replaced by $k_{s 0}$ ) the governing equations may be written as

$$
\begin{aligned}
\ddot{X} & =-\dot{Y} r_{1}\left[\epsilon \dot{X}-\left(\frac{\phi_{1}}{r_{1} \dot{Y}}+\phi_{2}\right) \frac{r_{1}}{r_{2}} \dot{Y}\right] \\
\ddot{Y} & =-\dot{Y} r_{1}\left[\epsilon \dot{Y}+c_{1} \epsilon^{2}\left(\frac{\phi_{1}}{r_{1} \dot{Y}}+\phi_{2}\right) \frac{r_{1}}{r_{2}} \dot{X}\right] \\
\ddot{Z} & =-1-\epsilon r_{1} \dot{Y} \dot{Z} .
\end{aligned}
$$

The variable spin is represented through the term involving $\phi_{1}$ and this appears at leading order in the $\ddot{X}$ equation. Hence, variable spin is expected to have a significant effect on the $X$ motion. However, $\phi_{1}$ and $X$ only appear in the $\ddot{Y}$ and $\ddot{Z}$ equations at second order and so it is expected that variable spin has little effect on the $Y, Z$ motion.

Using a standard perturbation, to $\mathcal{O}(\epsilon)$ the solutions are

$$
X=\left(\phi_{1}+\phi_{2}\right) \frac{T^{2}}{2}+\epsilon\left[\frac{c_{2} W^{2}\left(\phi_{1}+2 \phi_{2}\right) T^{2}}{4}-\left(\phi_{1}\left(2+c_{2} W\right)+\phi_{2}\left(3+2 c_{2} W\right)\right) \frac{T^{3}}{6}+\frac{c_{2}\left(\phi_{1}+2 \phi_{2}\right) T^{4}}{24}\right]
$$

$$
Y=T-\epsilon \frac{T^{2}}{2}, \quad Z=W T-\frac{T^{2}}{2}+\epsilon\left[-\frac{W T^{2}}{2}+\frac{T^{3}}{6}\right]
$$



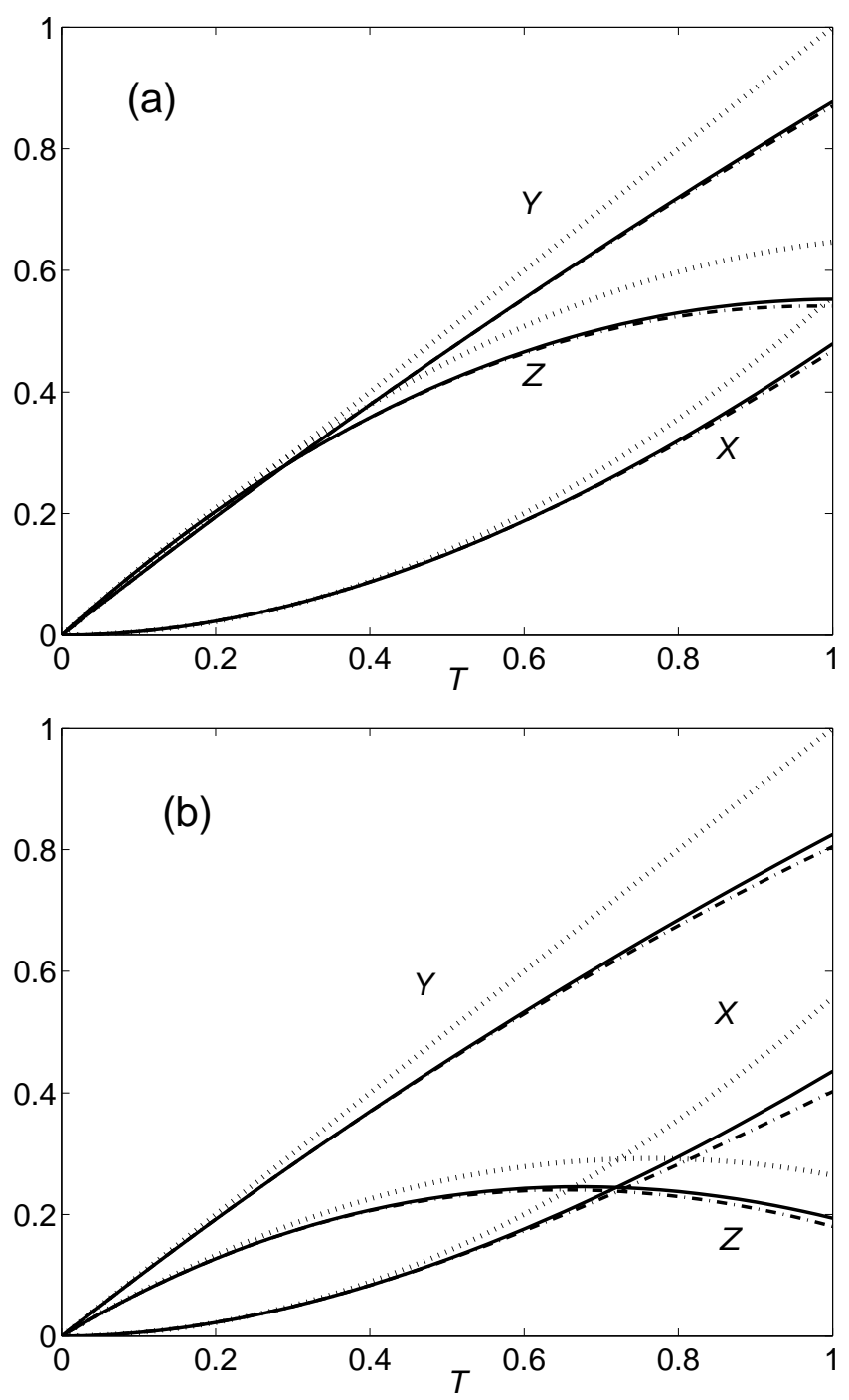

Fig. 6 Non-dimensional trajectories of $X, Y$ and $Z$ against $T$ for variable $C_{s}$ (with $\omega=$ 50): full numerical solution (solid line), $\mathcal{O}\left(\epsilon^{0}\right)$ solution (dotted line) and $\mathcal{O}(\epsilon)$ solution (dot-dashed line): a) $L_{2}=20$, b) $L_{2}=30 \mathrm{~m}$.

Noting that $k_{l}=0$ leads to $c_{3}=c_{4}=0$ and then the $Y$ and $Z$ solutions above correspond to equations (21-23) to $\mathcal{O}(\epsilon)$ while the $X$ solution differs from equation (21) at leading order.

Figure 6 shows a comparison of numerical and perturbation solutions for the model including variable spin. Figure 6 a) has the same initial conditions as used in Figure 3 but now with $C_{s}$ varying according to equation (38) and 
$\omega=50 \mathrm{rad} / \mathrm{s}$. The solid line is the numerical solution and the broken lines indicate various levels of perturbation solution. The $X$ curve shows that at the end of the trajectory the ball has moved slightly less than 0.6 non-dimensional units, which corresponds to a dimensional distance $0.6 k_{s 0} L_{2}^{2} \approx 2.59 \mathrm{~m}$. Since the dimensional swerve is proportional to $L_{2}$, in Figure $6 \mathrm{~b}$ ) the same kick is shown but now taken from $30 \mathrm{~m}$ away. The change in $L_{2}$ results in an increase in $\epsilon=k_{d} L_{2}$ and so it is expected that the perturbation solution will lose accuracy. This is clear from the figure, however the $\mathcal{O}(\epsilon)$ solution is still relatively accurate (accuracy could be intcreased by finding the $\mathcal{O}\left(\epsilon^{2}\right)$ term). The dimensional swerve at the end of the kick is now around $4.86 \mathrm{~m}$. This is almost double that of the $20 \mathrm{~m}$ kick and results from the approximately quadratic dependence on distance.

In Figure 7a) four curves corresponding to $\omega=30,40,50,60 \mathrm{rad} / \mathrm{s}$ and $L_{2}=30 \mathrm{~m}$ are plotted (with other conditions as in Figure 3). As expected increasing $\omega$ increases the lateral motion such that $X(1)$ ranges from 0.298 to 0.44 as $\omega$ increases from 30 to 60 (and dimensionally from $2.9 \mathrm{~m}$ to $4.3 \mathrm{~m}$ ). Neglecting variable drag, equation (29) indicates that the increase should be approximately linear $x \propto k_{s}$. Including the variation in drag coefficient, with a cut-off, makes the increase nonlinear. For example, as $\omega$ increases from 30 to $40 X(1)$ increases by 0.052 (dimensionally $0.5 \mathrm{~m}$ ), but as increasing $\omega$ from 50 to $60 X(1)$ increases by only 0.04 (dimensionally $0.4 \mathrm{~m}$ ). Increasing $\omega$ further will make very little difference to the motion. This may be understood through Figure $7 \mathrm{~b}$ ) which shows the variation of $C_{s}$ through the trajectory: with $\omega=30$ or 40 the value of $C_{s}$ is always increasing. When $\omega=50$ the value of $C_{s}$ becomes constant near the end of the flight and for $\omega=60$ it is constant half of the time. Consequently any kick with $\omega>60$ will provide a similar result as that with $\omega=60$. In carrying out this study the question arose as to what exactly is being presented by different research groups when plotting $C_{s}-S p$ curves. In particular it appears unclear how $S p=R \omega /|\boldsymbol{v}|$ is interpreted. The experiments of Asai et al. and Passmore et al. $[1,15]$ involve a ball fixed in a wind tunnel where both $\omega$ and $\boldsymbol{v}$ remain constant and so $S p$ takes a single value for each experiment. The drag and lateral force are measured directly, which then allows calculation of drag coefficients through the definitions of equation (2). More 'realistic experiments' involve actually kicking the ball in a controlled environment and then calculating coefficients by matching the data to some mathematical model. For example, Bray \& Kerwin [2] track a ball's flight with two cameras. The model equations are solved and iterated, using the drag coefficients as fitting parameters, until a good fit to the experimental data is achieved. By this method constant values for forward and lateral drag coefficients are determined for a given kick. However, since these values come from fitting to the full trajectory, where $S p$ is an increasing function, the coefficients must be some form of average for the specific kick. A similar technique is employed by Carré et al. [5] where drag coefficients are also averages, but plots are against the initial value of $S p$ (thus avoiding the confusion in the variation of $S p$ ). Goff and Carré $[7,8]$ fit drag coefficients to minimise the least-squares error between a numerical solution and experimental data but to 

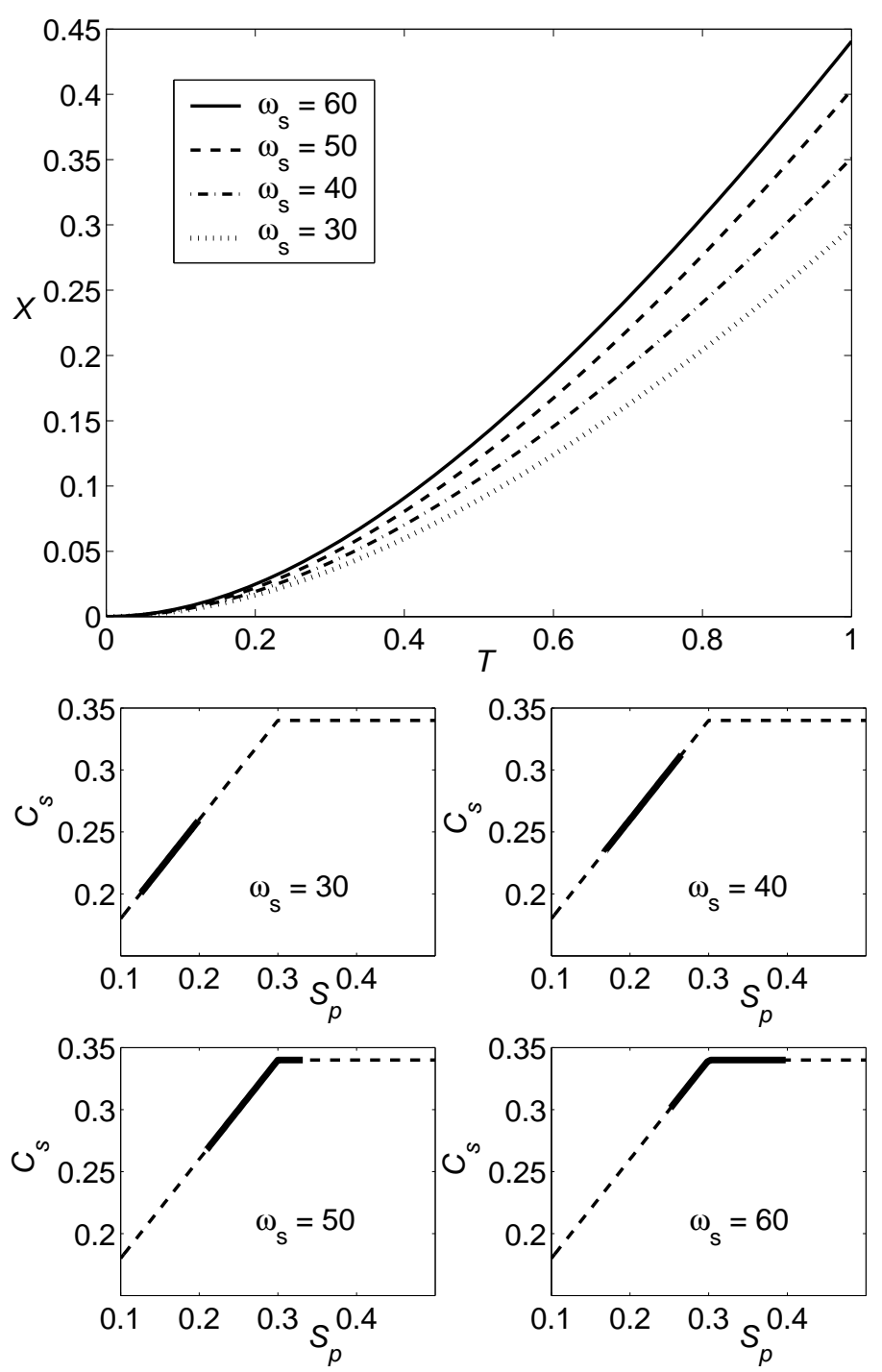

Fig. 7 a) Non-dimensional trajectories of $x$ against $t$ for variable $\left.C_{s}, b\right)$ corresponding plots of $C_{s}$ against $S_{p}$, dashed line represents equation (38) the thick solid line is the variation of $C_{s}$ over the trajectory.

reduce variation in $S p$ they limit data to the first $0.07 \mathrm{~s}$ of flight. Griffiths et al. [9] use an accurate ball tracking method but then calculate coefficients using the formula of Wesson [16] which assumes constant drag. Consequently, the various data presented may show actual $C_{s}$ and $S p$ values or may be average values over a given trajectory (with different methods of averaging). Figure 8 presents two sets of curves, the bottom curves correspond to variable $C_{s}$ with 
$L_{2}=30 \mathrm{~m}$. The dashed line represents the ball trajectory up to $t=1.655 \mathrm{~s}$ (to end at $y \approx 30 \mathrm{~m}$ ) the solid line ends at $t=0.693 \mathrm{~s}$ (around $y=15 \mathrm{~m}$ ). The top curves use a constant value of $C_{s}=0.307$ which was chosen to match the variable $C_{s}$ result after $1.655 \mathrm{~s}$. The graph is presented dimensionally, since the non-dimensional scaling depends on the choice of $C_{s}$. All other conditions are the same as in the $\omega=50 \mathrm{rad} / \mathrm{s}$ case of Figure 7 . The important feature here is that for times not equal to $1.655 \mathrm{~s}$ the constant $C_{s}$ curve has a different amount of swerve which indicates that if an average value is used for $C_{s}$ then its value will vary with the distance of the kick (in the figure the $15 \mathrm{~m}$ kicks show a difference of $10 \mathrm{~cm}$ in swerve). That is, if all other conditions are fixed fitting a constant $C_{s}$ value to experimental data will lead to range of $C_{s}$ values for kicks taken over different distances.

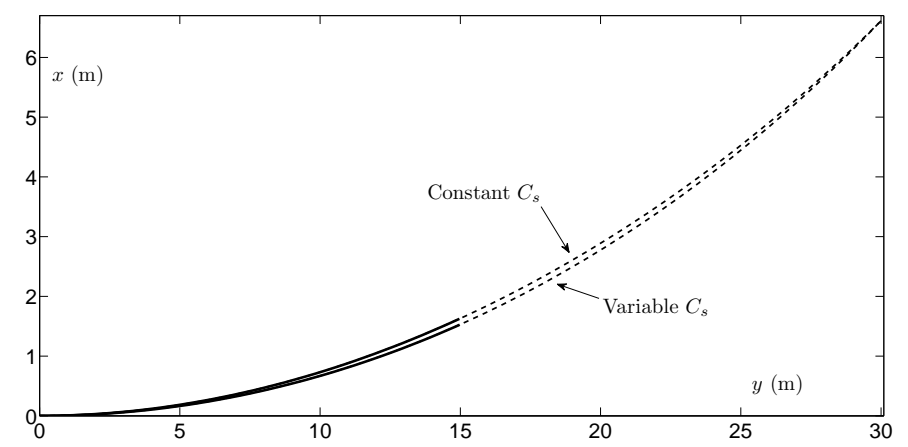

Fig. 8 Comparison of trajectories for varying and constant $C_{s}$.

\section{Discussion}

In this study the three-dimensional equations of motion for a football in flight have been studied. The numerical solution is trivial but this does not show the dependence on the problem parameters. Consequently a perturbation solution was developed which proved highly accurate when compared to the numerical solution. In deriving this solution the scaling was based on the assumption that footballers impart more lateral than top spin. This could easily be changed, to study a golf ball for example, where top-spin dominates. The analysis allowed a number of observations to be made about the ball's motion.

The analytical approximations show that at leading order the lateral motion is proportional to the lateral drag coefficient $k_{s}$ and the distance $(v t)^{2}$. This indicates why free kicks taken from a large distance show greater swerve. To first order in the small parameter, the motion in each direction is at most described by a quartic equation in time. Experimental studies often approx- 
imate data with a polynomial. For example, Carré et al. [5] show excellent agreement with data assuming $x$ varies quadratically in $t$. This is in agreement with this study's leading order result $x \approx k_{s}(v t)^{2} / 2$ and so will capture the dominant behaviour and small time solution well. Bray and Kerwin [2] assume a quartic dependence for $x, y, z$ : since a quartic contains more coefficients it can more accurately fit the data. This form agrees with the first order expression. For this reason the results presented here have not been compared with experimental data: numerous studies have shown that the data may be well approximated by quadratic or quartic functions. Since the results take this form equally good agreement can be obtained by simply choosing appropriate coefficients (and so inferring values for the drag coefficients). In fact this shows that in a sense the governing equations may be considered as rather forgiving in that an inaccurate model will still allow good agreement with experimental data. Missing out terms, for example by studying only the two-dimensional motion, will lead to the same form of solution as the three-dimensional system but the polynomial coefficients will be different. As a consequence a numerical study of a slightly incorrect set of governing equations will be able to provide excellent agreement with experimental data but the drag coefficients calculated from this solution will differ from the true values. To illustrate this, equations $(29,30)$ are combined to determine an expression for $x$ as a function of $y$, accurate to $\mathcal{O}(\epsilon)$,

$$
x=\frac{k_{s} y^{2}}{2}\left[1+\left\{\frac{w^{2}}{v^{2}}-\frac{2 g w y}{3 v^{3}}+\frac{g^{2} y^{2}}{6 v^{4}}\right\}\right],
$$

where the $\mathcal{O}(\epsilon)$ terms are in the curly brackets. At leading order, the wellknown approximation that $x$ varies quadratically with $y$ is observed. As time, and so $y$, increases the $\mathcal{O}(\epsilon)$ term grows in importance and causes the flight to deviate from the quadratic and this deviation is described by a quartic. The correct coefficient of $y^{2}$ in the above equation is $k_{s}\left(1+w^{2} / v^{2}\right) / 2$. Given a polynomial fit to experimental data and values of the initial velocities $v, w$ it is then possible to calculate $k_{s}$ by simply comparing the appropriate polynomial coefficients. However, if the mathematical model neglects the $z$ motion (so setting $w=0$ in (50)) then the prediction for $k_{s}$ will be a factor of approximately $\left(1+w^{2} / v^{2}\right)$ greater than the true value.

Obviously then, care should be taken in the choice of model to extract drag coefficients from three dimensional experimental data. If the ball is launched in a gravitational field (rather difficult to avoid) with side spin then the full three-dimensional equations should be used, since the vertical velocity has a marked effect on the lateral motion. The exception to this rule is when the ball is launched with zero velocity in the $x$ direction and with only top (or bottom) spin, then a two-dimensional study in the $y-z$ plane will suffice. Given that $C_{s}$ arises from the same effect as $C_{l}$ only one of these quantities actually needs to be determined and so the two-dimensional experiment would provide all necessary information.

In the final section the effect of varying $C_{s}$ (or $C_{l}$ ) with spin during the flight was investigated. Numerical studies generally invoke a constant value, 
often chosen to provide the best fit against data. The results in this study show that varying $C_{s}$ does make a difference to the predicted trajectories. While it is possible to choose an average constant value this value will vary with the length of the trajectory. Since the drag coefficient is a function of the ball and air properties, not the distance of the flight, this is clearly a physically unrealistic result. An issue that arose during this part of the study involved the interpretation of spin. To determine $C_{s}$ in terms of $S p$ an experiment may be carried out where the trajectory of the football is tracked by some motion capturing equipment. Comparison with the results from a mathematical model then leads to a value of $C_{s}$ for a stated $S p$ for each experiment. However, since $S p$ actually increases through the flight it is not clear what is meant by the quoted value. Exceptions to this rule are experiments carried out with a fixed ball in a wind tunnel, when $S p$ can be kept constant, see [1,15] for example.

The well-known jump in the drag coefficient, which can lead to reverse swing in other sports, was not considered in this paper since a football's flight normally occurs at values of the Reynolds number above the transition [11]. However, it is worth noting that the transition occurs at higher $R e$ for smoother balls. Given the reduction in air density with altitude (and so $R e$ ) this could be an important factor in the motion of a smooth ball in high altitude stadiums, such as in Johannesburg. In fact, during the 2010 World Cup where the Jabulani ball was used (a smooth ball with no seams), there was much criticism over the balls seemingly erratic flight. For example, the England coach Fabio Capello stated that the ball behaved 'oddly at altitude', Brazilian striker Fabiano stated that it unpredictably changed direction when traveling through the air, see [13].

The question of whether the choice of ball can provide an advantage for a team, and in particular does altitude make a difference, is now discussed. From equation (29) it may be seen that the swerve in the $x$ direction is proportional to $k_{s}=\rho A C_{s} /(2 m)$. The value of the air density $\rho$ decreases with altitude. In high altitude stadiums, such as Johannesburg at $1800 \mathrm{~m}, \rho \approx 1.04 \mathrm{~kg} / \mathrm{m}^{3}$ is approximately $20 \%$ lower than at sea-level, $\rho \approx 1.29 \mathrm{~kg} / \mathrm{m}^{3}$. Hence a team accustomed to playing at sea level will expect approximately $20 \%$ more swerve. To illustrate the difference more clearly in Figure 9 three trajectories are shown for identical kicks with $\rho=1.04,1.19,1.29 \mathrm{~kg} / \mathrm{m}^{3}$ (the latter two values being appropriate for Madrid and Barcelona). The initial value of $v=36 \mathrm{~m} / \mathrm{s}$ and $L_{2}=35 \mathrm{~m}$, the drag coefficient is taken as constant, $C_{s}=0.34$, other conditions are the same as in earlier figures. If the ball is initially kicked at a level with a goalpost then a velocity in the $x$ direction of $-15.16 \mathrm{~m} / \mathrm{s}$ just leads to a goal at sea/level, when $\rho=1.29$. (The numerical solution is used here since $\dot{x}$ was not included in the perturbation and also this free kick rather tests the perturbation.) The same kick taken in Madrid misses the goal by around $1.3 \mathrm{~m}$ while in Johannesburg it misses by around $3.3 \mathrm{~m}$. Hörzer et al. [11] found similar behaviour through their numerical simulations.

The advantage of the analytical solution presented in this paper is that while it shows not only explicitly the effect of air density but it also shows the dependence on other variables. For example, since swerve is proportional 
to $\rho A C_{s}$ the change in the ball's behaviour due to altitude can equally well be made by changing the drag coefficient. The trajectory for $\rho=1.29$ in Figure 9 could be made identical to the $\rho=1.04$ curve by setting $C_{s}=$ $1.04 \times 0.34 / 1.29 \approx 0.274$. This is currently an important issue for football manufacturers (prompted by the 2010 South African world cup) who wish to develop balls that show the same behaviour at different altitudes.

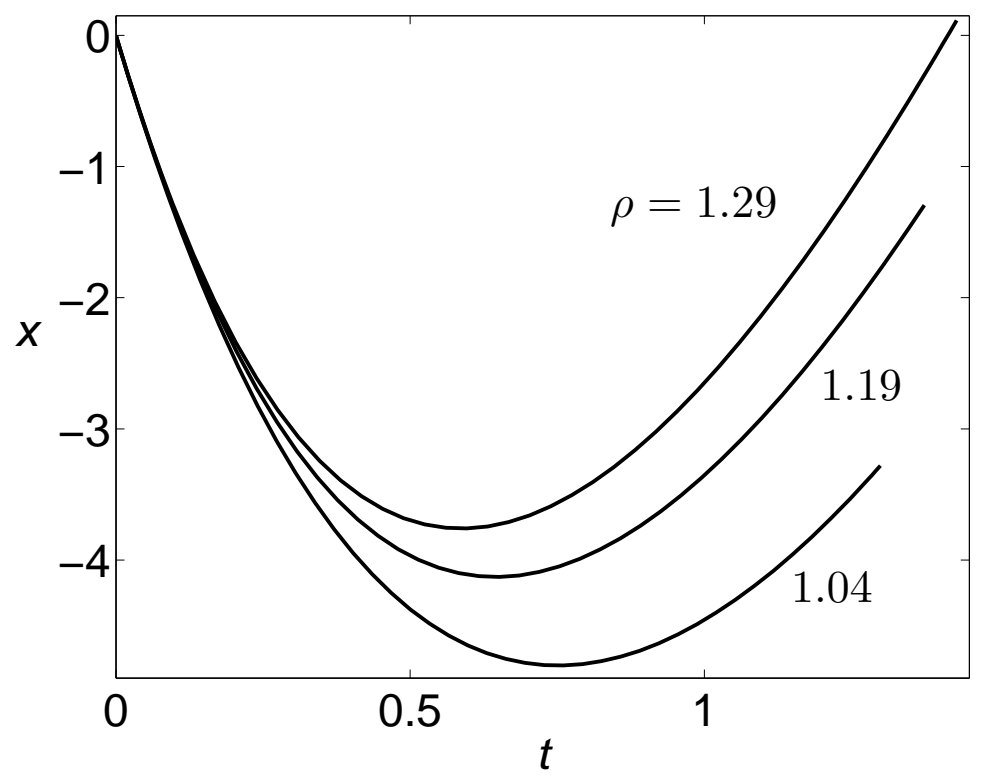

Fig. 9 Difference in trajectories at different altitudes.

Finally, there remains the question of whether this analysis can make a practical difference. The analytical solution makes clear the factors affecting the balls flight. It shows that coastal teams expect more swerve than teams at high altitude, decreasing air density or the balls roughness both act to reduce swerve. Consequently, after the meeting held in Johannesburg in January 2011 it was suggested that Bidvest Wits use a smooth ball in matches since this will behave in the manner furthest from that expected by teams that normally play at a lower altitude. When practising for away games they should use a rough ball. Coastal teams could apply the opposite argument and choose rough balls when playing high altitude teams. Of course one cannot say what factors affect a team's change in fortunes, but it should be noted that before the meeting, Wits had not won in 8 consecutive games. In their first home match after the meeting, played against a coastal team, they won 6-0. Up to the end of the 2011 season they subsequently lost only one home game which was a cup tie, where the home team do not provide the ball. Unfortunately they did not fare so well at away games. 
Acknowledgements The research of TGM was supported by the Marie Curie International Reintegration Grant Industrial applications of moving boundary problems, grant no. FP7-256417 and Ministerio de Ciencia e Innovación grant MTM2010-17162. SLM acknowledges the support of the Mathematics Applications Consortium for Science and Industry (MACSI, www.macsi.ul.ie) funded by the Science Foundation Ireland Mathematics Initiative Grant 06/MI/005.

\section{References}

1. Asai, K. Seo, O. Kobayashi, and R. Sakashita, Fundamental aerodynamics of the soccer ball. Sports Eng. 10, 101110, (2007)

2. K. Bray and D. Kerwin. Modelling the flight of a soccer ball in a direct free kick, J. Sports Sci., 21(2), 75-85, (2003)

3. K. Bray and D. Kerwin. Simplified flight equations for a spinning soccer ball. Science and Football V: Proc. 5th world congress on sci. and football, (2005)

4. M. J. Carré, S. R. Goodwill, and S. J. Haake, Understanding the effect of seams on the aerodynamics of an association football, J. Mech. Eng. Sci. 219, 657666, (2005)

5. M.J. Carré, T. Asai, T. Akatsuka and S.J Hakke. The Curve Kick of a Football ll: Flight through the air, Sports Engng., 193-200, (2002)

6. Dupeux G. , Le Goff A., Qur D. and Clanet C. The spinning ball spiral. New J. Physics 12, 2010, doi:10.1088/1367-2630/12/9/093004

7. J.E. Goff and M.J. Carré. Trajectory analysis of a soccer ball, Am. J. Phys., 77(11), 1020-1027, (2009)

8. J.E. Goff and M.J. Carré. Soccer ball lift coefficients via trajectory analysis, Eur. J. Phys., 31, 775-784, (2010)

9. I. Griffiths, C. Evans and N. Griffiths. Tracking the flight of a spinning football in three dimensions, Meas. Sci. Technol., 16, 2056-2065, (2005)

10. The history of soccer, http://www. soccerballworld.com/History.htm\#Early, last accessed 4th May 2011

11. S. Hörzer, C. Fuchs, R. Gastinger, A. Sabo, L. Mehnem, J. Martinek and M. Reichel. Simulation of spinning soccer ball trajectories influenced by altitude, 8th Conference of the Int. Sports Engng. Assoc. (ISEA), Procedia Engng. 2, 2461-2466, (2010)

12. Jabulani, http://www.jabulaniball.com/, last accessed 4th May 2011

13. Adidas Jabulani, http://en.wikipedia.org/wiki/Adidas_Jabulani, last accessed 4th May 2011

14. L. Oggiano and L. Sætran. Aerodynamics of modern soccer balls, Procedia Engng, 2, 2473-2479, (2010)

15. M. Passmore, S. Tuplin, A. Spencer and R. Jones. Experimental studies of the aerodynamics of spinning and stationary footballs, Proc. Inst. Mech. Engineers, Part C: J Mech. Engng. Sci., 222(2), 195-205, (2008)

16. J. Wesson The science of soccer. IoP Pub. Ltd., (2002) 